\title{
Turbocharger Flow Computations with the Space-Time Isogeometric Analysis (ST-IGA)
}

\author{
Kenji Takizawa ${ }^{\mathrm{a}, *}$, Tayfun E. Tezduyar ${ }^{\mathrm{b}}$, Yuto Otoguro ${ }^{\mathrm{a}}$, Takuya Terahara ${ }^{\mathrm{a}}$, \\ Takashi Kuraishi $^{\mathrm{a}}$, Hitoshi Hattori ${ }^{\mathrm{a}}$ \\ ${ }^{a}$ Department of Modern Mechanical Engineering, Waseda University \\ 1-6-1 Nishi-Waseda, Shinjuku-ku, Tokyo 169-8050, Japan \\ ${ }^{b}$ Mechanical Engineering, Rice University - MS 321 \\ 6100 Main Street, Houston, TX 77005, USA
}

\begin{abstract}
We focus on turbocharger computational flow analysis with a method that possesses higher accuracy in spatial and temporal representations. In the method we have developed for this purpose, we use a combination of i) the SpaceTime Variational Multiscale (ST-VMS) method, which is a stabilized formulation that also serves as a turbulence model, ii) the ST Slip Interface (ST-SI) method, which maintains high-resolution representation of the boundary layers near spinning solid surfaces by allowing in a consistent fashion slip at the interface between the mesh covering a spinning surface and the mesh covering the rest of the domain, and iii) the Isogeometric Analysis (IGA), where we use NURBS basis functions in space and time. The basis functions are spatially higher-order in all representations, and temporally higher-order in representation of the solid-surface and mesh motions. The ST nature of the method gives us higher-order accuracy in the flow solver, and when combined with temporally higher-order basis functions, a more accurate representation of the surface motion, and a mesh motion consistent with that. The spatially higher-order basis functions give us again higher-order accuracy in the flow solver, a more accurate, in some parts exact, representation of the surface geometry, and better representation in evaluating the second-order spatial derivatives. Using NURBS
\end{abstract}

\footnotetext{
${ }^{*}$ Corresponding author. Tel.: +81-3-5286-2143.

Email address: Kenji.Takizawa@tafsm.org (Kenji Takizawa)
}

Preprint submitted to Computers \& Fluids

February 26, 2016 
basis functions with a complex geometry is not trivial, however, once we generate the mesh, the computational efficiency is substantially increased. We focus on the turbine part of a turbocharger, but our method can also be applied to the compressor part and thus can be extended to the full turbocharger. Keywords: Turbocharger, turbine, Space-Time Variational Multiscale method, ST-VMS, ST Slip Interface method, ST-SI, Isogeometric Analysis, IGA, higher-order functions

\section{Introduction}

The Space-Time Variational Multiscale (ST-VMS) method $<1 ; 2>$ is the VMS version of the Deforming-Spatial-Domain/Stabilized ST (DSD/SST) method $<3 ; 4 ; 5>$. The VMS components are from the residual-based VMS (RBVMS) method given in $<6 ; 7 ; 8 ; 9>$. The stabilization components of the original DSD/SST method are the Streamline-Upwind/Petrov-Galerkin (SUPG) $<10>$ and Pressure-Stabilizing/Petrov-Galerkin (PSPG) $<3>$ stabilizations, and for that the method is now also called "ST-SUPS" (the acronym was coined in $<11>$ ).

The ST-VMS method was successfully applied to wind-turbine aerodynamics $<12 ; 13 ; 14 ; 11 ; 15 ; 16 ; 17 ; 18 ; 19>$, flapping-wing aerodynamics $<20 ; 21 ; 22$; $11 ; 23 ; 24 ; 25 ; 16 ; 17 ; 26>$, cardiovascular fluid mechanics $<27 ; 28 ; 24 ; 29 ; 16 ; 17$; $30 ; 31>$, spacecraft aerodynamics $<32 ; 33>$, thermo-fluid analysis of ground vehicles and their tires $<34>$, and flow-driven string dynamics in turbomachinery $<35>$.

The ST methods involve more computational cost per time step than a semidiscrete method, but they have a number of desirable features $($ see $<1 ; 2>$ ). One of those desirable features is being able to use higher-order basis functions in time, including the non-uniform rational B-spline (NURBS) basis functions, which have been used very effectively as spatial basis functions (see, for example, $<36 ; 37 ; 38 ; 39 ; 40 ; 41 ; 12 ; 42 ; 43 ; 44 ; 45 ; 11 ; 46 ; 47 ; 48 ; 18 ; 49 ; 50 ; 51 ; 52 ; 53 ; 54$; $55 ; 56>)$. The ST context provides higher-order accuracy in the flow solver, and when combined with temporally higher-order basis functions, a more accurate 
representation of the surface motion, and a mesh motion consistent with that. It also provides more efficiency in temporal representation of the motion and deformation of the volume meshes, and better efficiency in remeshing. These have been demonstrated in a number of 3D computations, specifically, flapping-wing aerodynamics $<20 ; 21 ; 22 ; 11 ; 23 ; 24 ; 25 ; 16 ; 17 ; 26>$, separation aerodynamics of spacecraft $<32>$, wind-turbine aerodynamics $<15 ; 16 ; 17 ; 18 ; 19>$, and thermo-fluid analysis of ground vehicles and their tires $<34>$. The ST framework and NURBS in time also enable, with the "ST-C" method, extracting a continuous representation from the computed data and, in large-scale computations, efficient data compression $<57 ; 34>$.

In computation of flow problems where a subdomain contains a spinning solid surface, as in a turbine, we want the mesh covering the subdomain containing the spinning solid surface to spin with it so that we maintain the high-resolution representation of the boundary layers. For that, something needs to be done at the interfaces between the subdomains. The ST computation of this class of problems was first accomplished with the Shear-Slip Mesh Update Method $(\mathrm{SSMUM})<58 ; 59 ; 60>$, which was introduced in $<58 ; 59>$ and named "SSMUM" in $<60>$. The ST/NURBS Mesh Update Method (STNMUM), which was introduced in $<20 ; 21 ; 22\rangle$ and named in $\langle 15\rangle$, is more general and relatively simpler to use. It was used in $<15>$ in ST-VMS computation of flow past a wind-turbine rotor, and the tower was included in the computational model. In the STNMUM, NURBS basis functions are used for the temporal representation of the spinning motion, mesh motion and also in remeshing. The motion of the structure is represented by quadratic temporal NURBS basis functions, with sufficient number of temporal patches for one rotation. This enables us to represent the circular paths associated with the spinning motion exactly and, with a "secondary mapping" $<1 ; 20 ; 2 ; 11>$, specify a constant angular velocity corresponding to the invariant speeds along those paths.

The method introduced most recently in the ST context for flow problems with spinning solid surfaces is the ST Slip Interface (ST-SI) method. The starting point in the development of the ST-SI method was the version of the ALE- 
VMS method designed for computations with "sliding interfaces" $<45 ; 61>$, which has been used successfully in a number of computations with spinning structures $<45 ; 50 ; 47 ; 49 ; 61>$. In the ST-SI method, interface terms similar to those in the ALE-VMS version are added to the ST-VMS formulation to account for the compatibility conditions for the velocity and stress. In addition to having a high-resolution representation of the boundary layers near the spinning structure, because the ST framework allows NURBS functions in temporal representation of the structure motion, the ST-SI method has exact representation of the circular paths associated with the spinning.

The RBVMS method was tested in $<8>$ in conjunction with the Isogeometric Analysis (IGA) $<36>$, and the test results showed that using quadratic or cubic NURBS basis functions in space significantly improves the solution accuracy compared to the finite element analysis with trilinear basis functions. Quadratic NURBS basis functions in space were incorporated into the ST-VMS method first time in $\langle 1\rangle$, and some $2 \mathrm{D}$ test computations with an airfoil geometry were also presented in $\langle 1\rangle$. Here we incorporate the higher-order NURBS basis functions in space into the ST-VMS method in the 3D context. The spatially higher-order basis functions give us higher-order accuracy in the flow solver, a more accurate, in some parts exact, representation of the surface geometry, and better representation in evaluating the second-order spatial derivatives. Being able to include those second-order derivatives enables us to properly model the second-order derivatives in the turbulence-modeling terms of the ST-VMS formulation, and this gives us a better spatial discretization in terms of the consistency of the method. In addition, a NURBS mesh that represents a solid surface exactly can be refined further for higher-resolution representation of the flow field near the solid surface without changing the surface geometry. Using NURBS basis functions with a complex geometry is not trivial, however, once we generate the mesh, the computational efficiency is substantially increased.

In this article, we focus on turbocharger computational flow analysis with a method we introduce here by combining the ST-VMS and ST-SI methods and the IGA. We will call the combined method the "Space-Time Isogeometric Anal- 
ysis (ST-IGA)." In this work, we consider the turbine part of a turbocharger, but our approach can also be applied to the compressor part and thus can be extended to the full turbocharger. We describe the ST-VMS and ST-SI methods in Section 2. In Section 3, we present the computations, first a 3D test computation with a circular-pipe flow, and then the computation for the turbine. The concluding remarks are given in Section 4.

\section{Method}

This section is a condensed version of the directly related parts of the formulations presented in $<19>$. We first describe the ST-VMS method:

$$
\begin{aligned}
& \int_{Q_{n}} \mathbf{w}^{h} \cdot \rho\left(\frac{\partial \mathbf{u}^{h}}{\partial t}+\mathbf{u}^{h} \cdot \nabla \mathbf{u}^{h}-\mathbf{f}^{h}\right) \mathrm{d} Q+\int_{Q_{n}} \boldsymbol{\varepsilon}\left(\mathbf{w}^{h}\right): \boldsymbol{\sigma}\left(\mathbf{u}^{h}, p^{h}\right) \mathrm{d} Q \\
& -\int_{\left(P_{n}\right)_{\mathrm{h}}} \mathbf{w}^{h} \cdot \mathbf{h}^{h} \mathrm{~d} P+\int_{Q_{n}} q^{h} \boldsymbol{\nabla} \cdot \mathbf{u}^{h} \mathrm{~d} Q+\int_{\Omega_{n}}\left(\mathbf{w}^{h}\right)_{n}^{+} \cdot \rho\left(\left(\mathbf{u}^{h}\right)_{n}^{+}-\left(\mathbf{u}^{h}\right)_{n}^{-}\right) \mathrm{d} \Omega \\
& +\sum_{e=1}^{\left(n_{\mathrm{el}}\right)_{n}} \int_{Q_{n}^{e}} \frac{\tau_{\mathrm{SUPS}}}{\rho}\left[\rho\left(\frac{\partial \mathbf{w}^{h}}{\partial t}+\mathbf{u}^{h} \cdot \boldsymbol{\nabla} \mathbf{w}^{h}\right)+\nabla q^{h}\right] \cdot \mathbf{r}_{\mathrm{M}}\left(\mathbf{u}^{h}, p^{h}\right) \mathrm{d} Q \\
& +\sum_{e=1}^{\left(n_{\mathrm{el}}\right)_{n}} \int_{Q_{n}^{e}} \nu_{\mathrm{LSIC}} \boldsymbol{\nabla} \cdot \mathbf{w}^{h} \rho r_{\mathrm{C}}\left(\mathbf{u}^{h}\right) \mathrm{d} Q \\
& -\sum_{e=1}^{\left(n_{\mathrm{el}}\right)_{n}} \int_{Q_{n}^{e}} \tau_{\mathrm{SUPS}} \mathbf{w}^{h} \cdot\left(\mathbf{r}_{\mathrm{M}}\left(\mathbf{u}^{h}, p^{h}\right) \cdot \nabla \mathbf{u}^{h}\right) \mathrm{d} Q \\
& -\sum_{e=1}^{\left(n_{\mathrm{el}}\right)_{n}} \int_{Q_{n}^{e}} \frac{\tau_{\mathrm{SUPS}}^{2}}{\rho} \mathbf{r}_{\mathrm{M}}\left(\mathbf{u}^{h}, p^{h}\right) \cdot\left(\boldsymbol{\nabla} \mathbf{w}^{h}\right) \cdot \mathbf{r}_{\mathrm{M}}\left(\mathbf{u}^{h}, p^{h}\right) \mathrm{d} Q=0
\end{aligned}
$$

where

$$
\begin{aligned}
\mathbf{r}_{\mathrm{M}}\left(\mathbf{u}^{h}, p^{h}\right) & =\rho\left(\frac{\partial \mathbf{u}^{h}}{\partial t}+\mathbf{u}^{h} \cdot \nabla \mathbf{u}^{h}-\mathbf{f}^{h}\right)-\nabla \cdot \boldsymbol{\sigma}\left(\mathbf{u}^{h}, p^{h}\right), \\
r_{\mathrm{C}}\left(\mathbf{u}^{h}\right) & =\nabla \cdot \mathbf{u}^{h}
\end{aligned}
$$

are the residuals of the momentum equation and incompressibility constraint. Here, $\rho, \mathbf{u}, p, \mathbf{f}, \boldsymbol{\sigma}, \boldsymbol{\varepsilon}$, and $\mathbf{h}$ are the density, velocity, pressure, external force, stress tensor, strain rate tensor, and the traction specified at the boundary. The test functions associated with the velocity and pressure are $\mathbf{w}$ and $q$. A 
superscript " $h$ " indicates that the function is coming from a finite-dimensional space. The symbol $Q_{n}$ represents the ST slice between time levels $n$ and $n+1$, $\left(P_{n}\right)_{\mathrm{h}}$ is the part of the lateral boundary of that slice associated with the traction boundary condition $\mathbf{h}$, and $\Omega_{n}$ is the spatial domain at time level $n$. The superscript " $e$ " is the ST element counter, and $n_{\mathrm{el}}$ is the number of ST elements. The functions are discontinuous in time at each time level, and the superscripts "-" and "+" indicate the values of the functions just below and just above the time level. There are various ways of defining the stabilization parameters $\tau_{\mathrm{SUPS}}$ and $\nu_{\text {LSIC }}$. See $<4 ; 5 ; 15 ; 34 ; 19>$ for the stabilization parameter definitions used here. For more ways of calculating the stabilization parameters in finite element computation of flow problems, see $<62 ; 63 ; 64 ; 65 ; 66 ; 67 ; 68 ; 69 ; 70$; $71 ; 72 ; 73 ; 74 ; 75 ; 76 ; 77 ; 78 ; 79 ; 80 ; 81 ; 82 ; 83>$.

The expression for $\mathbf{r}_{\mathrm{M}}\left(\mathbf{u}^{h}, p^{h}\right)$ includes second derivatives of the velocity. For linear basis functions these terms vanish, and for bilinear and trilinear basis functions they are grossly underrepresented. This means that $\mathbf{r}_{\mathrm{M}}\left(\mathbf{u}^{h}, p^{h}\right)$ does not explicitly depend on the Reynolds number. When we use quadratic or higher-order basis functions, on the other hand, the term is nonzero and therefore explicit dependence of the residual on the Reynolds number is taken into account.

In describing the ST-SI formulation (see $<19>$ ), we use the labels "Side A" and "Side B" to represent the two sides of the SI. In the ST-SI version of the formulation given by Eq. (1), we have added boundary terms corresponding to the SI. The boundary terms for the two sides are first added separately, using test functions $\mathbf{w}_{\mathrm{A}}^{h}$ and $q_{\mathrm{A}}^{h}$ and $\mathbf{w}_{\mathrm{B}}^{h}$ and $q_{\mathrm{B}}^{h}$. Then, putting together the terms added to each side, the complete set of terms added becomes

$$
\begin{aligned}
& -\int_{\left(P_{n}\right)_{\mathrm{SI}}}\left(q_{\mathrm{B}}^{h} \mathbf{n}_{\mathrm{B}}-q_{\mathrm{A}}^{h} \mathbf{n}_{\mathrm{A}}\right) \cdot \frac{1}{2}\left(\mathbf{u}_{\mathrm{B}}^{h}-\mathbf{u}_{\mathrm{A}}^{h}\right) \mathrm{d} P \\
& -\int_{\left(P_{n}\right)_{\mathrm{SI}}} \rho \mathbf{w}_{\mathrm{B}}^{h} \cdot \frac{1}{2}\left(\left(\mathcal{F}_{\mathrm{B}}^{h}-\left|\mathcal{F}_{\mathrm{B}}^{h}\right|\right) \mathbf{u}_{\mathrm{B}}^{h}-\left(\mathcal{F}_{\mathrm{B}}^{h}-\left|\mathcal{F}_{\mathrm{B}}^{h}\right|\right) \mathbf{u}_{\mathrm{A}}^{h}\right) \mathrm{d} P \\
& -\int_{\left(P_{n}\right)_{\mathrm{SI}}} \rho \mathbf{w}_{\mathrm{A}}^{h} \cdot \frac{1}{2}\left(\left(\mathcal{F}_{\mathrm{A}}^{h}-\left|\mathcal{F}_{\mathrm{A}}^{h}\right|\right) \mathbf{u}_{\mathrm{A}}^{h}-\left(\mathcal{F}_{\mathrm{A}}^{h}-\left|\mathcal{F}_{\mathrm{A}}^{h}\right|\right) \mathbf{u}_{\mathrm{B}}^{h}\right) \mathrm{d} P
\end{aligned}
$$




$$
\begin{aligned}
& +\int_{\left(P_{n}\right)_{\mathrm{SI}}}\left(\mathbf{n}_{\mathrm{B}} \cdot \mathbf{w}_{\mathrm{B}}^{h}+\mathbf{n}_{\mathrm{A}} \cdot \mathbf{w}_{\mathrm{A}}^{h}\right) \frac{1}{2}\left(p_{\mathrm{B}}^{h}+p_{\mathrm{A}}^{h}\right) \mathrm{d} P \\
& -\int_{\left(P_{n}\right)_{\mathrm{SI}}}\left(\mathbf{w}_{\mathrm{B}}^{h}-\mathbf{w}_{\mathrm{A}}^{h}\right) \cdot\left(\hat{\mathbf{n}}_{\mathrm{B}} \cdot \mu\left(\varepsilon\left(\mathbf{u}_{\mathrm{B}}^{h}\right)+\varepsilon\left(\mathbf{u}_{\mathrm{A}}^{h}\right)\right)\right) \mathrm{d} P \\
& -\int_{\left(P_{n}\right)_{\mathrm{SI}}} \hat{\mathbf{n}}_{\mathrm{B}} \cdot \mu\left(\varepsilon\left(\mathbf{w}_{\mathrm{B}}^{h}\right)+\varepsilon\left(\mathbf{w}_{\mathrm{A}}^{h}\right)\right) \cdot\left(\mathbf{u}_{\mathrm{B}}^{h}-\mathbf{u}_{\mathrm{A}}^{h}\right) \mathrm{d} P \\
& +\int_{\left(P_{n}\right)_{\mathrm{SI}}} \frac{\mu C}{h}\left(\mathbf{w}_{\mathrm{B}}^{h}-\mathbf{w}_{\mathrm{A}}^{h}\right) \cdot\left(\mathbf{u}_{\mathrm{B}}^{h}-\mathbf{u}_{\mathrm{A}}^{h}\right) \mathrm{d} P,
\end{aligned}
$$

where

$$
\begin{aligned}
\mathcal{F}_{\mathrm{B}}^{h} & =\mathbf{n}_{\mathrm{B}} \cdot\left(\mathbf{u}_{\mathrm{B}}^{h}-\mathbf{v}_{\mathrm{B}}^{h}\right), \\
\mathcal{F}_{\mathrm{A}}^{h} & =\mathbf{n}_{\mathrm{A}} \cdot\left(\mathbf{u}_{\mathrm{A}}^{h}-\mathbf{v}_{\mathrm{A}}^{h}\right), \\
h & =\min \left(h_{\mathrm{B}}, h_{\mathrm{A}}\right), \\
h_{\mathrm{B}} & =2\left(\sum_{\alpha=1}^{n_{\text {ent }}} \sum_{a=1}^{n_{\text {ens }}}\left|\mathbf{n}_{\mathrm{B}} \cdot \nabla N_{a}^{\alpha}\right|\right)^{-1} \text { (for Side B), } \\
h_{\mathrm{A}} & =2\left(\sum_{\alpha=1}^{n_{\text {ent }}} \sum_{a=1}^{n_{\text {ens }}}\left|\mathbf{n}_{\mathrm{A}} \cdot \nabla N_{a}^{\alpha}\right|\right)^{-1} \text { (for Side A), } \\
\hat{\mathbf{n}}_{\mathrm{B}} & =\frac{\mathbf{n}_{\mathrm{B}}-\mathbf{n}_{\mathrm{A}}}{\left\|\mathbf{n}_{\mathrm{B}}-\mathbf{n}_{\mathrm{A}}\right\|} .
\end{aligned}
$$

Here, $\left(P_{n}\right)_{\mathrm{SI}}$ is the SI in the ST domain, $\mathbf{n}$ is the unit normal vector, $\mathbf{v}$ is the mesh velocity, $n_{\mathrm{ens}}$ and $n_{\mathrm{ent}}$ are the number of spatial and temporal element nodes, $N_{a}^{\alpha}$ is the basis function associated with spatial and temporal nodes $a$ and $\alpha$, and $C$ is a nondimensional penalty constant. Usually $C=1$ is large enough for stability.

A number of remarks were provided in $<19>$ to explain the added terms and to comment on related interpretations. We refer the reader interested in such details to $<19>$.

On solid surfaces where we prefer to have weakly-imposed Dirichlet conditions for the fluid, we use the ST-SI version where the SI is between the fluid and solid domains. That version was obtained in $<19>$ by starting with the terms added to Side B and replacing the Side A velocity with the velocity $\mathbf{g}^{h}$ coming from the solid domain. Then the terms added to Eq. (1) to represent the weakly-imposed Dirichlet conditions become 


$$
\begin{aligned}
& -\int_{\left(P_{n}\right)_{\mathrm{SI}}} q_{\mathrm{B}}^{h} \mathbf{n}_{\mathrm{B}} \cdot \mathbf{u}_{\mathrm{B}}^{h} \mathrm{~d} P-\int_{\left(P_{n}\right)_{\mathrm{SI}}} \rho \mathbf{w}_{\mathrm{B}}^{h} \cdot \mathcal{F}_{\mathrm{B}}^{h} \mathbf{u}_{\mathrm{B}}^{h} \mathrm{~d} P+\int_{\left(P_{n}\right)_{\mathrm{SI}}} q_{\mathrm{B}}^{h} \mathbf{n}_{\mathrm{B}} \cdot \mathbf{g}^{h} \mathrm{~d} P \\
& +\int_{\left(P_{n}\right)_{\mathrm{SI}}} \rho \mathbf{w}_{\mathrm{B}}^{h} \cdot \frac{1}{2}\left(\left(\mathcal{F}_{\mathrm{B}}^{h}+\left|\mathcal{F}_{\mathrm{B}}^{h}\right|\right) \mathbf{u}_{\mathrm{B}}^{h}+\left(\mathcal{F}_{\mathrm{B}}^{h}-\left|\mathcal{F}_{\mathrm{B}}^{h}\right|\right) \mathbf{g}^{h}\right) \mathrm{d} P \\
& -\int_{\left(P_{n}\right)_{\mathrm{SI}}} \mathbf{w}_{\mathrm{B}}^{h} \cdot\left(\mathbf{n}_{\mathrm{B}} \cdot \boldsymbol{\sigma}_{\mathrm{B}}^{h}\right) \mathrm{d} P-\int_{\left(P_{n}\right)_{\mathrm{SI}}} \mathbf{n}_{\mathrm{B}} \cdot 2 \mu \varepsilon\left(\mathbf{w}_{\mathrm{B}}^{h}\right) \cdot\left(\mathbf{u}_{\mathrm{B}}^{h}-\mathbf{g}^{h}\right) \mathrm{d} P \\
& +\int_{\left(P_{n}\right)_{\mathrm{SI}}} \frac{\mu C}{h_{\mathrm{B}}} \mathbf{w}_{\mathrm{B}}^{h} \cdot\left(\mathbf{u}_{\mathrm{B}}^{h}-\mathbf{g}^{h}\right) \mathrm{d} P .
\end{aligned}
$$

\section{Computations}

We first present a 3D test computation with a circular-pipe flow, and then the computation for the turbine part of a turbocharger.

\subsection{Circular-pipe flow}

The Reynolds number is $1.1 \times 10^{5}$. The pipe length is $80 D$, where $D$ is the diameter. Figure 1 shows the NURBS mesh at the inflow boundary, which is the same at all cross-sections. The number of control points is 659,126 , and the

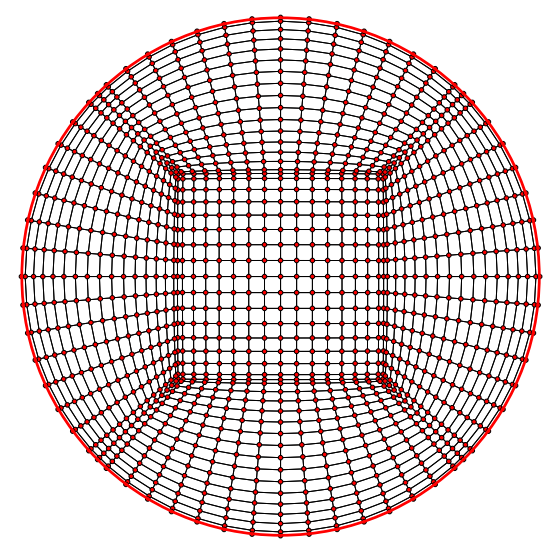

Figure 1: Circular-pipe flow. NURBS control mesh. The red circle shown with the mesh is the actual boundary.

number of elements is 562,500 . We note that for the first layer of elements near 
the pipe wall, the thickness in the normal direction is rather large compared to a typical finite element mesh used for this class of problems.

We use the stabilization parameters given in $\langle 15\rangle$, which mostly originate from those given in $\langle 4 ; 5\rangle$. The Dirichlet boundary conditions at the pipe wall are strongly-imposed. The number of nonlinear iterations per time step is 3, and the number of GMRES iterations per nonlinear iteration is 700 . We compute until we reach the steady state. We calculate an average friction factor by using the expression $\frac{8\left(\tau_{\mathrm{w}}\right)_{\mathrm{AXI}}}{\rho U^{2}}$, where $\left(\tau_{\mathrm{w}}\right)_{\mathrm{AXI}}$ is the axial wall shear stress averaged over the pipe, leaving the inflow and outflow effects out, and $U$ is the volumetric flow rate divided by the pipe cross-sectional area.

The friction factor obtained from our computation is $1.72 \times 10^{-2}$, and the experimental value from a textbook is $1.70 \times 10^{-2}$, which is also for a smooth pipe. Although the NURBS mesh resolution in the normal direction near the pipe wall is much lower compared to a typical finite element mesh used for this class of problems, the solution obtained is very close to the experimental value.

\subsection{Turbine}

Figure 2 shows the turbine geometry and the NURBS mesh for the rotor.

Figure 3 shows the NURBS mesh for the volute. The interface between the rotor

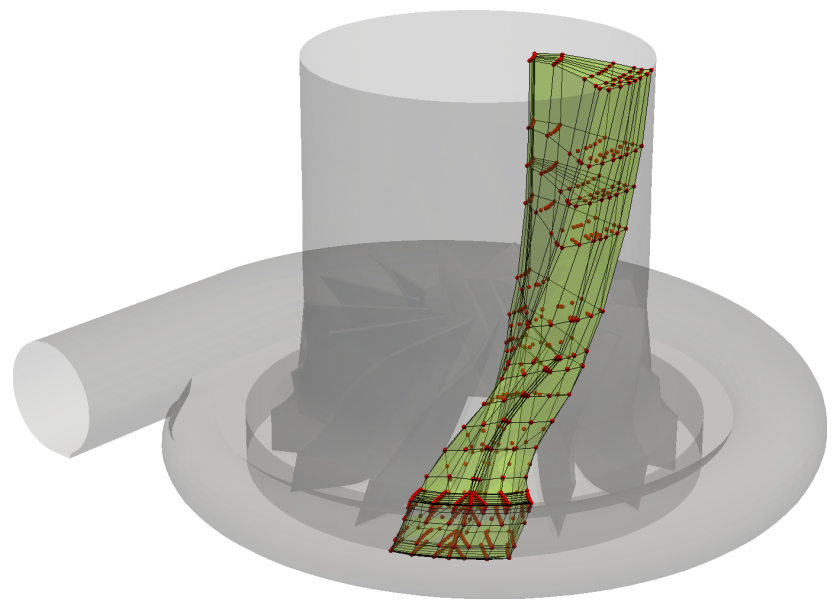

Figure 2: Turbine. Geometry and 1/13 of the NURBS mesh for the rotor. 


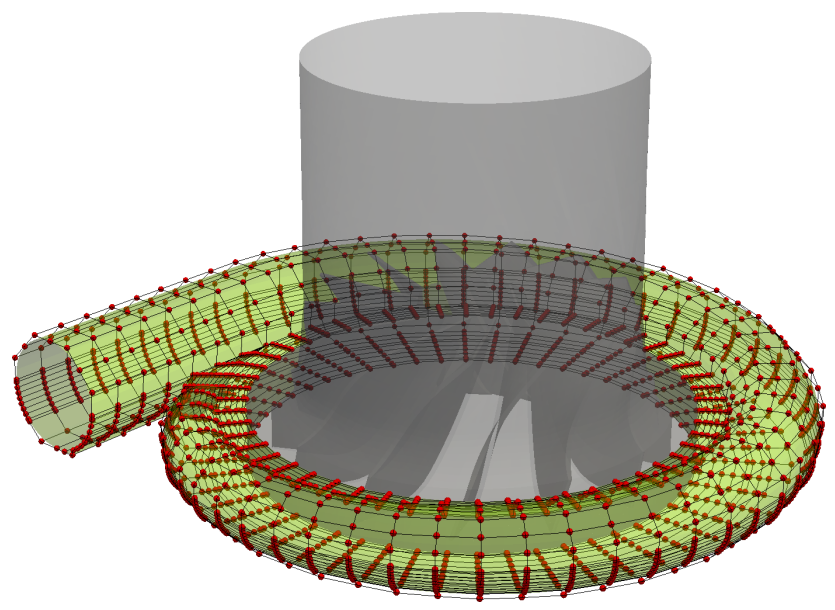

Figure 3: Turbine. NURBS mesh for the volute.

and volute meshes is taken as an SI of the ST-SI method, and so is the interface between the rotor and outflow-segment meshes. In addition, the interface where the narrow part of the volute connects to the wide part is also taken as an SI, and this avoids the difficulty of matching NURBS patches. Although, the mesh is rather coarse, the geometry is represented quite well.

The rotor speed is 50,000 rpm and the inflow rate is $0.01035 \mathrm{~m}^{3} / \mathrm{s}$. We use the stabilization parameters given in $\langle 19\rangle$, which mostly originate from those given in $\langle 4 ; 5 ; 34\rangle$. The Dirichlet boundary conditions on all solid surfaces are weakly-imposed. The number of time steps for a full rotation is 180 . The number of nonlinear iterations per time step is 3, and the number of GMRES iterations per nonlinear iteration is 300 .

Figures 4 and 5 show the results from this computation. Figure 4 is the velocity magnitude at the end of the 7 th rotation, and Figure 5 is the turbine efficiency during the 7 th rotation. The efficiency is defined as

$$
\eta=\frac{P}{K},
$$

where $P$ is the power extracted from the turbine, and

$$
K=\int_{\Gamma_{\mathrm{INF}}}\left(\frac{1}{2} \rho\|\mathbf{u}\|+p-p_{\text {OUTF }}\right) \mathrm{d} \Gamma .
$$


Here $\Gamma_{\text {INF }}$ is the inflow boundary, and $p_{\text {OUTF }}$ is the outflow pressure. Typically $p_{\text {OUTF }}$ is set indirectly to zero through the zero-stress condition at the outflow boundary, and is therefore set to zero in the calculation of $K$ here.

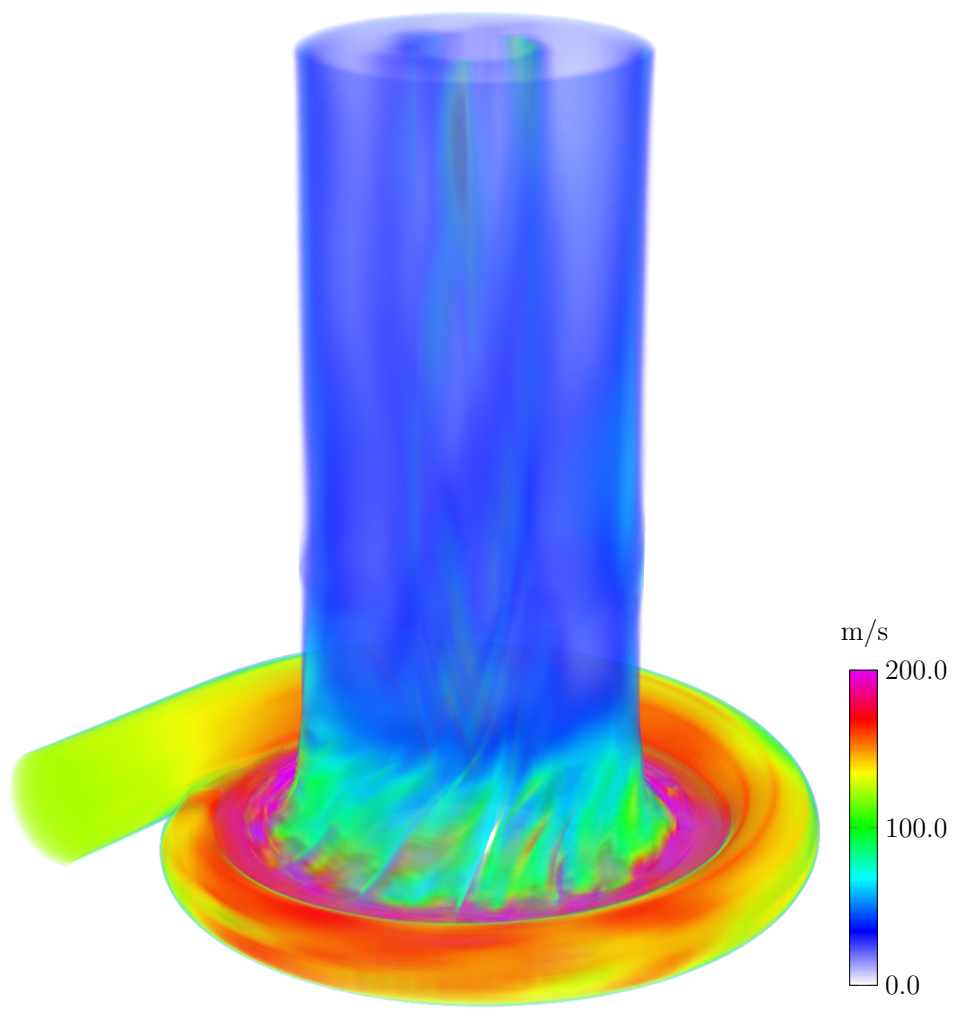

Figure 4: Turbine. Velocity magnitude at the end of the 7 th rotation.

\section{Concluding Remarks}

We have presented a turbocharger computational flow analysis method that possesses higher accuracy in spatial and temporal representations. The components of the method, which we call "ST-IGA," are the ST-VMS and ST-SI methods and the IGA. The ST-VMS method is a stabilized formulation that also serves as a turbulence model. The ST-SI method maintains high-resolution representation of the boundary layers near spinning solid surfaces by allowing 


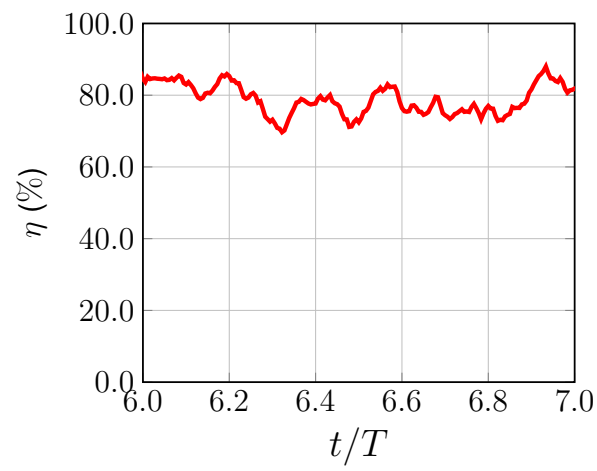

Figure 5: Turbine. Efficiency during the 7 th rotation; $T$ is the rotation period.

in a consistent fashion slip at the interface between the mesh covering a spinning surface and the mesh covering the rest of the domain. In the IGA, we use NURBS basis functions in space and time; the basis functions are spatially higher-order in all representations, and temporally higher-order in representation of the solid-surface and mesh motions. The ST nature of the method gives us higher-order accuracy in the flow solver, and in combination with temporally higher-order basis functions, a more accurate representation of the surface motion, and a mesh motion consistent with that. The spatially higher-order basis functions give us again higher-order accuracy in the flow solver and a more accurate, in some parts exact, representation of the surface geometry. They also lead to a better representation in evaluating the second-order spatial derivatives, and that enables us to properly model the second-order derivatives in the turbulence-modeling terms of the ST-VMS formulation. We presented first a 3D test computation with a circular-pipe flow, and then the computation for the turbine part of a turbocharger. With the pipe flow computation, we showed that a quadratic NURBS mesh, even with a relatively low mesh resolution in the normal direction near the pipe wall, yields a friction factor that is very close to the experimental value. With the turbine computation, we showed that we are able to successfully generate NURBS volume meshes for complex problems such as the turbine of a turbocharger and obtain reasonable solutions even with rel- 
atively coarse meshes. Although we focused in this article on the turbine part of the turbocharger, our method can also be applied to the compressor part and thus can be extended to the full turbocharger. Overall, we demonstrated the potential of the the ST-IGA for computational analysis of challenging flow problems, including turbomachinery flows.

\section{Acknowledgement}

This work was supported (first, third, fourth, fifth, and sixth authors) in part by Grant-in-Aid for Young Scientists (B) 24760144 from Japan Society for the Promotion of Science (JSPS); Grant-in-Aid for Scientific Research (S) 26220002 from the Ministry of Education, Culture, Sports, Science and Technology of Japan (MEXT); Council for Science, Technology and Innovation (CSTI), Cross-ministerial Strategic Innovation Promotion Program (SIP), "Innovative Combustion Technology" (Funding agency: JST); and Rice-Waseda research agreement. This work was also supported (second author) in part by ARO Grant W911NF-12-1-0162.

[1] K. Takizawa and T.E. Tezduyar, "Multiscale space-time fluid-structure interaction techniques", Computational Mechanics, 48 (2011) 247-267, doi: 10.1007/s00466-011-0571-z.

[2] K. Takizawa and T.E. Tezduyar, "Space-time fluid-structure interaction methods", Mathematical Models and Methods in Applied Sciences, 22 (2012) 1230001, doi: 10.1142/S0218202512300013.

[3] T.E. Tezduyar, "Stabilized finite element formulations for incompressible flow computations", Advances in Applied Mechanics, 28 (1992) 1-44, doi: 10.1016/S0065-2156(08)70153-4.

[4] T.E. Tezduyar, "Computation of moving boundaries and interfaces and stabilization parameters", International Journal for Numerical Methods in Fluids, 43 (2003) 555-575, doi: 10.1002/fld.505. 
[5] T.E. Tezduyar and S. Sathe, "Modeling of fluid-structure interactions with the space-time finite elements: Solution techniques", International Journal for Numerical Methods in Fluids, 54 (2007) 855-900, doi: 10.1002/fld.1430.

[6] T.J.R. Hughes, "Multiscale phenomena: Green's functions, the Dirichletto-Neumann formulation, subgrid scale models, bubbles, and the origins of stabilized methods", Computer Methods in Applied Mechanics and Engineering, 127 (1995) 387-401.

[7] T.J.R. Hughes, A.A. Oberai, and L. Mazzei, "Large eddy simulation of turbulent channel flows by the variational multiscale method", Physics of Fluids, 13 (2001) 1784-1799.

[8] Y. Bazilevs, V.M. Calo, J.A. Cottrell, T.J.R. Hughes, A. Reali, and G. Scovazzi, "Variational multiscale residual-based turbulence modeling for large eddy simulation of incompressible flows", Computer Methods in Applied Mechanics and Engineering, 197 (2007) 173-201.

[9] Y. Bazilevs and I. Akkerman, "Large eddy simulation of turbulent TaylorCouette flow using isogeometric analysis and the residual-based variational multiscale method", Journal of Computational Physics, 229 (2010) 34023414 .

[10] A.N. Brooks and T.J.R. Hughes, "Streamline upwind/Petrov-Galerkin formulations for convection dominated flows with particular emphasis on the incompressible Navier-Stokes equations", Computer Methods in Applied Mechanics and Engineering, 32 (1982) 199-259.

[11] Y. Bazilevs, K. Takizawa, and T.E. Tezduyar, Computational FluidStructure Interaction: Methods and Applications. Wiley, February 2013, ISBN 978-0470978771.

[12] Y. Bazilevs, M.-C. Hsu, I. Akkerman, S. Wright, K. Takizawa, B. Henicke, T. Spielman, and T.E. Tezduyar, "3D simulation of wind turbine rotors 
at full scale. Part I: Geometry modeling and aerodynamics", International Journal for Numerical Methods in Fluids, 65 (2011) 207-235, doi: 10.1002/fld.2400.

[13] K. Takizawa, B. Henicke, T.E. Tezduyar, M.-C. Hsu, and Y. Bazilevs, "Stabilized space-time computation of wind-turbine rotor aerodynamics", Computational Mechanics, 48 (2011) 333-344, doi: 10.1007/s00466-0110589-2.

[14] K. Takizawa, B. Henicke, D. Montes, T.E. Tezduyar, M.-C. Hsu, and Y. Bazilevs, "Numerical-performance studies for the stabilized space-time computation of wind-turbine rotor aerodynamics", Computational Mechanics, 48 (2011) 647-657, doi: 10.1007/s00466-011-0614-5.

[15] K. Takizawa, T.E. Tezduyar, S. McIntyre, N. Kostov, R. Kolesar, and C. Habluetzel, "Space-time VMS computation of wind-turbine rotor and tower aerodynamics", Computational Mechanics, 53 (2014) 1-15, doi: 10.1007/s00466-013-0888-x.

[16] K. Takizawa, Y. Bazilevs, T.E. Tezduyar, M.-C. Hsu, O. Øiseth, K.M. Mathisen, N. Kostov, and S. McIntyre, "Engineering analysis and design with ALE-VMS and space-time methods", Archives of Computational Methods in Engineering, 21 (2014) 481-508, doi: 10.1007/s11831-014-91130.

[17] K. Takizawa, "Computational engineering analysis with the new-generation space-time methods", Computational Mechanics, 54 (2014) 193-211, doi: 10.1007/s00466-014-0999-z.

[18] Y. Bazilevs, K. Takizawa, T.E. Tezduyar, M.-C. Hsu, N. Kostov, and S. McIntyre, "Aerodynamic and FSI analysis of wind turbines with the ALE-VMS and ST-VMS methods", Archives of Computational Methods in Engineering, 21 (2014) 359-398, doi: 10.1007/s11831-014-9119-7. 
[19] K. Takizawa, T.E. Tezduyar, H. Mochizuki, H. Hattori, S. Mei, L. Pan, and K. Montel, "Space-time VMS method for flow computations with slip interfaces (ST-SI)", Mathematical Models and Methods in Applied Sciences, 25 (2015) 2377-2406, doi: 10.1142/S0218202515400126.

[20] K. Takizawa, B. Henicke, A. Puntel, T. Spielman, and T.E. Tezduyar, "Space-time computational techniques for the aerodynamics of flapping wings", Journal of Applied Mechanics, 79 (2012) 010903, doi: 10.1115/1.4005073.

[21] K. Takizawa, B. Henicke, A. Puntel, N. Kostov, and T.E. Tezduyar, "Space-time techniques for computational aerodynamics modeling of flapping wings of an actual locust", Computational Mechanics, 50 (2012) 743760, doi: 10.1007/s00466-012-0759-x.

[22] K. Takizawa, N. Kostov, A. Puntel, B. Henicke, and T.E. Tezduyar, "Space-time computational analysis of bio-inspired flapping-wing aerodynamics of a micro aerial vehicle", Computational Mechanics, 50 (2012) 761778, doi: 10.1007/s00466-012-0758-y.

[23] K. Takizawa, B. Henicke, A. Puntel, N. Kostov, and T.E. Tezduyar, "Computer modeling techniques for flapping-wing aerodynamics of a locust", Computers \& Fluids, 85 (2013) 125-134, doi: 10.1016/j.compfluid.2012.11.008.

[24] K. Takizawa, T.E. Tezduyar, A. Buscher, and S. Asada, "Space-time interface-tracking with topology change (ST-TC)", Computational Mechanics, 54 (2014) 955-971, doi: 10.1007/s00466-013-0935-7.

[25] K. Takizawa, T.E. Tezduyar, and N. Kostov, "Sequentially-coupled spacetime FSI analysis of bio-inspired flapping-wing aerodynamics of an MAV", Computational Mechanics, 54 (2014) 213-233, doi: 10.1007/s00466-0140980-x. 
[26] K. Takizawa, T.E. Tezduyar, and A. Buscher, "Space-time computational analysis of MAV flapping-wing aerodynamics with wing clapping", Computational Mechanics, 55 (2015) 1131-1141, doi: 10.1007/s00466-014-1095-0.

[27] K. Takizawa, K. Schjodt, A. Puntel, N. Kostov, and T.E. Tezduyar, "Patient-specific computer modeling of blood flow in cerebral arteries with aneurysm and stent", Computational Mechanics, 50 (2012) 675-686, doi: 10.1007/s00466-012-0760-4.

[28] K. Takizawa, K. Schjodt, A. Puntel, N. Kostov, and T.E. Tezduyar, "Patient-specific computational analysis of the influence of a stent on the unsteady flow in cerebral aneurysms", Computational Mechanics, 51 (2013) 1061-1073, doi: 10.1007/s00466-012-0790-y.

[29] K. Takizawa, Y. Bazilevs, T.E. Tezduyar, C.C. Long, A.L. Marsden, and K. Schjodt, "ST and ALE-VMS methods for patient-specific cardiovascular fluid mechanics modeling", Mathematical Models and Methods in Applied Sciences, 24 (2014) 2437-2486, doi: 10.1142/S0218202514500250.

[30] H. Suito, K. Takizawa, V.Q.H. Huynh, D. Sze, and T. Ueda, "FSI analysis of the blood flow and geometrical characteristics in the thoracic aorta", Computational Mechanics, 54 (2014) 1035-1045, doi: 10.1007/s00466-014$1017-1$.

[31] K. Takizawa, T.E. Tezduyar, A. Buscher, and S. Asada, "Space-time fluid mechanics computation of heart valve models", Computational Mechanics, 54 (2014) 973-986, doi: 10.1007/s00466-014-1046-9.

[32] K. Takizawa, D. Montes, M. Fritze, S. McIntyre, J. Boben, and T.E. Tezduyar, "Methods for FSI modeling of spacecraft parachute dynamics and cover separation", Mathematical Models and Methods in Applied Sciences, 23 (2013) 307-338, doi: 10.1142/S0218202513400058.

[33] K. Takizawa, D. Montes, S. McIntyre, and T.E. Tezduyar, "Spacetime VMS methods for modeling of incompressible flows at high 
Reynolds numbers", Mathematical Models and Methods in Applied Sciences, 23 (2013) 223-248, doi: 10.1142/s0218202513400022.

[34] K. Takizawa, T.E. Tezduyar, and T. Kuraishi, "Multiscale ST methods for thermo-fluid analysis of a ground vehicle and its tires", Mathematical Models and Methods in Applied Sciences, 25 (2015) 2227-2255, doi: 10.1142/S0218202515400072.

[35] H. Hattori, K. Takizawa, T.E. Tezduyar, K. Miyagawa, M. Nomi, M. Isono, H. Uchida, and M. Kawai, "Computational analysis of flow-driven string dynamics in a turbomachinery", in Proceedings of 13th Asian International Conference on Fluid Machinery, Paper No. AICFM13-154, Tokyo, Japan, (2015).

[36] T.J.R. Hughes, J.A. Cottrell, and Y. Bazilevs, "Isogeometric analysis: CAD, finite elements, NURBS, exact geometry, and mesh refinement", Computer Methods in Applied Mechanics and Engineering, 194 (2005) 4135-4195.

[37] Y. Bazilevs, V.M. Calo, Y. Zhang, and T.J.R. Hughes, "Isogeometric fluidstructure interaction analysis with applications to arterial blood flow", Computational Mechanics, 38 (2006) 310-322.

[38] Y. Bazilevs, V.M. Calo, T.J.R. Hughes, and Y. Zhang, "Isogeometric fluidstructure interaction: theory, algorithms, and computations", Computational Mechanics, 43 (2008) 3-37.

[39] Y. Bazilevs and T.J.R. Hughes, "NURBS-based isogeometric analysis for the computation of flows about rotating components", Computational Mechanics, 43 (2008) 143-150.

[40] Y. Bazilevs, J.R. Gohean, T.J.R. Hughes, R.D. Moser, and Y. Zhang, "Patient-specific isogeometric fluid-structure interaction analysis of thoracic aortic blood flow due to implantation of the Jarvik 2000 left ventricu- 
lar assist device", Computer Methods in Applied Mechanics and Engineering, 198 (2009) 3534-3550.

[41] Y. Bazilevs, M.-C. Hsu, D. Benson, S. Sankaran, and A. Marsden, "Computational fluid-structure interaction: Methods and application to a total cavopulmonary connection", Computational Mechanics, 45 (2009) 77-89.

[42] Y. Bazilevs, M.-C. Hsu, J. Kiendl, R. Wüchner, and K.-U. Bletzinger, "3D simulation of wind turbine rotors at full scale. Part II: Fluid-structure interaction modeling with composite blades", International Journal for $\mathrm{Nu}$ merical Methods in Fluids, 65 (2011) 236-253.

[43] Y. Bazilevs, M.-C. Hsu, K. Takizawa, and T.E. Tezduyar, "ALE-VMS and ST-VMS methods for computer modeling of wind-turbine rotor aerodynamics and fluid-structure interaction", Mathematical Models and Methods in Applied Sciences, 22 (2012) 1230002, doi: 10.1142/S0218202512300025.

[44] M.-C. Hsu, I. Akkerman, and Y. Bazilevs, "Wind turbine aerodynamics using ALE-VMS: Validation and role of weakly enforced boundary conditions", Computational Mechanics, 50 (2012) 499-511.

[45] M.-C. Hsu and Y. Bazilevs, "Fluid-structure interaction modeling of wind turbines: simulating the full machine", Computational Mechanics, 50 (2012) 821-833.

[46] Y. Bazilevs, K. Takizawa, and T.E. Tezduyar, "Challenges and directions in computational fluid-structure interaction", Mathematical Models and Methods in Applied Sciences, 23 (2013) 215-221, doi: 10.1142/S0218202513400010.

[47] A. Korobenko, M.-C. Hsu, I. Akkerman, J. Tippmann, and Y. Bazilevs, "Structural mechanics modeling and FSI simulation of wind turbines", Mathematical Models and Methods in Applied Sciences, 23 (2013) 249-272. 
[48] A. Korobenko, M.-C. Hsu, I. Akkerman, and Y. Bazilevs, "Aerodynamic simulation of vertical-axis wind turbines", Journal of Applied Mechanics, 81 (2013) 021011, doi: 10.1115/1.4024415.

[49] Y. Bazilevs, A. Korobenko, X. Deng, J. Yan, M. Kinzel, and J.O. Dabiri, "FSI modeling of vertical-axis wind turbines", Journal of Applied Mechanics, 81 (2014) 081006, doi: 10.1115/1.4027466.

[50] M.-C. Hsu, I. Akkerman, and Y. Bazilevs, "Finite element simulation of wind turbine aerodynamics: Validation study using NREL Phase VI experiment", Wind Energy, 17 (2014) 461-481.

[51] C.C. Long, M. Esmaily-Moghadam, A.L. Marsden, and Y. Bazilevs, "Computation of residence time in the simulation of pulsatile ventricular assist devices", Computational Mechanics, 54 (2014) 911-919, doi: 10.1007/s00466-013-0931-y.

[52] C.C. Long, A.L. Marsden, and Y. Bazilevs, "Shape optimization of pulsatile ventricular assist devices using FSI to minimize thrombotic risk", Computational Mechanics, 54 (2014) 921-932, doi: 10.1007/s00466-013-0967-z.

[53] M.-C. Hsu, D. Kamensky, Y. Bazilevs, M.S. Sacks, and T.J.R. Hughes, "Fluid-structure interaction analysis of bioprosthetic heart valves: significance of arterial wall deformation", Computational Mechanics, 54 (2014) 1055-1071, doi: 10.1007/s00466-014-1059-4.

[54] B. Augier, J. Yan, A. Korobenko, J. Czarnowski, G. Ketterman, and Y. Bazilevs, "Experimental and numerical FSI study of compliant hydrofoils", Computational Mechanics, 55 (2015) 1079-1090, doi: 10.1007/s00466-014-1090-5.

[55] Y. Bazilevs, A. Korobenko, J. Yan, A. Pal, S.M.I. Gohari, and S. Sarkar, "ALE-VMS formulation for stratified turbulent incompressible flows with applications", Mathematical Models and Methods in Applied Sciences, 25 (2015) 2349-2375, doi: 10.1142/S0218202515400114. 
[56] Y. Bazilevs, K. Takizawa, and T.E. Tezduyar, "New directions and challenging computations in fluid dynamics modeling with stabilized and multiscale methods", Mathematical Models and Methods in Applied Sciences, 25 (2015) 2217-2226, doi: 10.1142/S0218202515020029.

[57] K. Takizawa and T.E. Tezduyar, "Space-time computation techniques with continuous representation in time (ST-C)", Computational Mechanics, 53 (2014) 91-99, doi: 10.1007/s00466-013-0895-y.

[58] V. Kalro and T.E. Tezduyar, "Parallel finite element computation of 3D incompressible flows on MPPs", in W.G. Habashi, editor, Solution Techniques for Large-Scale CFD Problems, John Wiley \& Sons, 1995.

[59] T. Tezduyar, S. Aliabadi, M. Behr, A. Johnson, V. Kalro, and M. Litke, "Flow simulation and high performance computing", Computational Mechanics, 18 (1996) 397-412, doi: 10.1007/BF00350249.

[60] M. Behr and T. Tezduyar, "The Shear-Slip Mesh Update Method", Computer Methods in Applied Mechanics and Engineering, 174 (1999) 261-274, doi: 10.1016/S0045-7825(98)00299-0.

[61] Y. Bazilevs, A. Korobenko, X. Deng, and J. Yan, "Novel structural modeling and mesh moving techniques for advanced FSI simulation of wind turbines", International Journal for Numerical Methods in Engineering, 102 (2015) 766-783, doi: 10.1002/nme.4738.

[62] T.E. Tezduyar and D.K. Ganjoo, "Petrov-Galerkin formulations with weighting functions dependent upon spatial and temporal discretization: Applications to transient convection-diffusion problems", Computer Methods in Applied Mechanics and Engineering, 59 (1986) 49-71, doi: 10.1016/0045-7825(86)90023-X.

[63] G.J. Le Beau, S.E. Ray, S.K. Aliabadi, and T.E. Tezduyar, "SUPG finite element computation of compressible flows with the entropy and conserva- 
tion variables formulations", Computer Methods in Applied Mechanics and Engineering, 104 (1993) 397-422, doi: 10.1016/0045-7825(93)90033-T.

[64] T.E. Tezduyar, "Finite elements in fluids: Stabilized formulations and moving boundaries and interfaces", Computers 85 Fluids, 36 (2007) 191-206, doi: 10.1016/j.compfluid.2005.02.011.

[65] T.E. Tezduyar and M. Senga, "Stabilization and shock-capturing parameters in SUPG formulation of compressible flows", Computer Methods in Applied Mechanics and Engineering, 195 (2006) 1621-1632, doi: 10.1016/j.cma.2005.05.032.

[66] T.E. Tezduyar and M. Senga, "SUPG finite element computation of inviscid supersonic flows with YZ $\beta$ shock-capturing", Computers 8 Fluids, 36 (2007) 147-159, doi: 10.1016/j.compfluid.2005.07.009.

[67] T.E. Tezduyar, M. Senga, and D. Vicker, "Computation of inviscid supersonic flows around cylinders and spheres with the SUPG formulation and YZ $\beta$ shock-capturing", Computational Mechanics, 38 (2006) 469-481, doi: 10.1007/s00466-005-0025-6.

[68] T.E. Tezduyar and S. Sathe, "Enhanced-discretization selective stabilization procedure (EDSSP)", Computational Mechanics, 38 (2006) 456-468, doi: 10.1007/s00466-006-0056-7.

[69] A. Corsini, F. Rispoli, A. Santoriello, and T.E. Tezduyar, "Improved discontinuity-capturing finite element techniques for reaction effects in turbulence computation", Computational Mechanics, 38 (2006) 356-364, doi: 10.1007/s00466-006-0045-x.

[70] F. Rispoli, A. Corsini, and T.E. Tezduyar, "Finite element computation of turbulent flows with the discontinuity-capturing directional dissipation (DCDD)", Computers \& Fluids, 36 (2007) 121-126, doi: 10.1016/j.compfluid.2005.07.004. 
[71] T.E. Tezduyar, S. Ramakrishnan, and S. Sathe, "Stabilized formulations for incompressible flows with thermal coupling", International Journal for Numerical Methods in Fluids, 57 (2008) 1189-1209, doi: 10.1002/fld.1743.

[72] F. Rispoli, R. Saavedra, A. Corsini, and T.E. Tezduyar, "Computation of inviscid compressible flows with the V-SGS stabilization and YZ $\beta$ shock-capturing", International Journal for Numerical Methods in Fluids, 54 (2007) 695-706, doi: 10.1002/fld.1447.

[73] Y. Bazilevs, V.M. Calo, T.E. Tezduyar, and T.J.R. Hughes, "YZ $\beta$ discontinuity-capturing for advection-dominated processes with application to arterial drug delivery", International Journal for Numerical Methods in Fluids, 54 (2007) 593-608, doi: 10.1002/fld.1484.

[74] A. Corsini, C. Menichini, F. Rispoli, A. Santoriello, and T.E. Tezduyar, "A multiscale finite element formulation with discontinuity capturing for turbulence models with dominant reactionlike terms", Journal of Applied Mechanics, 76 (2009) 021211, doi: 10.1115/1.3062967.

[75] F. Rispoli, R. Saavedra, F. Menichini, and T.E. Tezduyar, "Computation of inviscid supersonic flows around cylinders and spheres with the V-SGS stabilization and YZ $\beta$ shock-capturing", Journal of Applied Mechanics, 76 (2009) 021209, doi: 10.1115/1.3057496.

[76] A. Corsini, C. Iossa, F. Rispoli, and T.E. Tezduyar, "A DRD finite element formulation for computing turbulent reacting flows in gas turbine combustors", Computational Mechanics, 46 (2010) 159-167, doi: 10.1007/s00466009-0441-0.

[77] M.-C. Hsu, Y. Bazilevs, V.M. Calo, T.E. Tezduyar, and T.J.R. Hughes, "Improving stability of stabilized and multiscale formulations in flow simulations at small time steps", Computer Methods in Applied Mechanics and Engineering, 199 (2010) 828-840, doi: 10.1016/j.cma.2009.06.019. 
[78] A. Corsini, F. Rispoli, and T.E. Tezduyar, "Stabilized finite element computation of NOx emission in aero-engine combustors", International Journal for Numerical Methods in Fluids, 65 (2011) 254-270, doi: 10.1002/fld.2451.

[79] A. Corsini, F. Rispoli, and T.E. Tezduyar, "Computer modeling of waveenergy air turbines with the SUPG/PSPG formulation and discontinuitycapturing technique", Journal of Applied Mechanics, 79 (2012) 010910, doi: 10.1115/1.4005060.

[80] A. Corsini, F. Rispoli, A.G. Sheard, and T.E. Tezduyar, "Computational analysis of noise reduction devices in axial fans with stabilized finite element formulations", Computational Mechanics, 50 (2012) 695-705, doi: $10.1007 / \mathrm{s} 00466-012-0789-4$.

[81] P.A. Kler, L.D. Dalcin, R.R. Paz, and T.E. Tezduyar, "SUPG and discontinuity-capturing methods for coupled fluid mechanics and electrochemical transport problems", Computational Mechanics, 51 (2013) 171185, doi: 10.1007/s00466-012-0712-z.

[82] A. Corsini, F. Rispoli, A.G. Sheard, K. Takizawa, T.E. Tezduyar, and P. Venturini, "A variational multiscale method for particle-cloud tracking in turbomachinery flows", Computational Mechanics, 54 (2014) 1191-1202, doi: 10.1007/s00466-014-1050-0.

[83] F. Rispoli, G. Delibra, P. Venturini, A. Corsini, R. Saavedra, and T.E. Tezduyar, "Particle tracking and particle-shock interaction in compressibleflow computations with the V-SGS stabilization and YZ $\beta$ shock-capturing", Computational Mechanics, 55 (2015) 1201-1209, doi: 10.1007/s00466-0151160-3. 